\title{
Active learning of introductory optics: interactive lecture demonstrations and optics magic tricks
}

David Sokoloff

David R. Sokoloff, "Active learning of introductory optics: interactive lecture demonstrations and optics magic tricks," Proc. SPIE 9665, Tenth International Topical Meeting on Education and Training in Optics and Photonics, 966516 (3 June 2007); doi: 10.1117/12.2207518

SPIE Event: Tenth International Topical Meeting on Education and Training in Optics and Photonics, 2007, Ottawa, Ontario, Canada 


\title{
Active Learning of Introductory Optics: Interactive Lecture Demonstrations and Optics Magic Tricks
}

\author{
David R. Sokoloff \\ Department of Physics, 1274 University of Oregon, Eugene, OR 97403-1274 USA \\ Telephone: 541-346-4755, e-mail: sokoloff@uoregon.edu
}

\begin{abstract}
Widespread physics education research has shown that most introductory physics students have difficulty learning essential optics concepts-even in the best of traditional courses, and that a well-designed active learning approach can remedy this. This active presentation will provide direct experience through audience participation with methods for promoting active involvement of students in the learning process. The focus will be on Interactive Lecture Demonstrations $(I L D s)^{1,2}$ - a learning strategy for large (and small) lectures, including the use of special Optics Magic Tricks. Sample ILD materials and instructions on how to do the tricks will be distributed.
\end{abstract}

Keywords: Introductory physics, active learning, introductory optics, introductory laboratory, lecture demonstrations, activity based physics, image formation

\section{Introduction}

There is considerable evidence that traditional approaches are ineffective in teaching physics concepts, including light and optics concepts. ${ }^{3,4}$ A major focus of the work at the University of Oregon and at the Center for Science and Mathematics Teaching (CSMT) at Tufts University has been on the development of active, discovery-based curricula like RealTime Physics labs ${ }^{4,5,6}$ and Interactive Lecture Demonstrations. ${ }^{1,2}$ Among the characteristics of these curricula are:

- Use of a learning cycle in which students are challenged to compare predictions-discussed with their peers in small groups - to observations of real experiments.

- Construction of students' knowledge from their own hands-on observations. Real observations of the physical world are the authority of knowledge.

- Confronting students with the differences between their observations and their beliefs.

- Observation of results from real experiments in understandable ways-often in real time with the support of microcomputer-based tools.

- $\quad$ Encouragement of collaboration and shared learning with peers.

- Laboratory work often used to learn basic concepts.

With the use of the learning cycle and the microcomputer-based tools it has been possible to bring about significant changes in the lecture and laboratory learning environments at a large number of universities, colleges and high schools without changing the lecture/laboratory structure of the introductory physics course. RealTime Physics and Interactive Lecture Demonstrations are described briefly below.

\section{RealTime Physics Active Learning Laboratories (RTP)}

RealTime Physics is series of lab modules for the introductory physics course that often use computer data acquisition tools to help students develop important physics concepts while acquiring vital laboratory skills. Besides data acquisition, computers are used for basic mathematical modeling, data analysis and some simulations. RTP labs use the learning cycle of prediction, observation and comparison. They have been demonstrated to enhance student learning of physics concepts. ${ }^{3,4}$ There are four RTP modules, Module 1: Mechanics, Module 2: Heat and

Tenth International Topical Meeting on Education and Training in Optics and Photonics, edited by Marc Nantel, Proc. of SPIE Vol. 9665, 966516 · @ 2007 SPIE, OSA, IEEE, ICO doi: $10.1117 / 12.2207518$ 
Thermodynamics, Module 3: Electric Circuits and Module 4: Light and Optics. ${ }^{5}$ Each lab includes a pre-lab preparation sheet to help students prepare, and a homework, designed to reinforce critical concepts and skills. A complete teachers' guide is available online for each module. This presentation will not include work with RTP labs.

\section{Interactive Lecture Demonstrations (ILDs)}

$I L D s$ are designed to enhance conceptual learning in large (and small) lectures. Real physics demonstrations are shown to students, who then make predictions about the outcomes on a prediction sheet, and collaborate with fellow students by discussing their predictions in small groups. Students then observe the results of the live demonstration (often displayed as real-time graphs using computer data acquisition tools), compare these results with their predictions, and attempt to explain the observed phenomena. Besides data acquisition, computers are used for interactive video analysis. The eight-step $I L D$ procedure incorporating this learning cycle is followed for each of the basic, single concept demonstrations in an $I L D$ sequence. $I L D s$ have been demonstrated to enhance student learning of physics concepts. ${ }^{2,3}$ Complete materials - including student sheets and teachers' guides—are available for most introductory physics topics. ${ }^{1}$

In this session, after an introduction to active learning, the eight-step Interactive Lecture Demonstration procedure will be illustrated through active audience participation. Examples will be drawn from the four sets of optics ILDs: Reflection and Refraction of Light, Image Formation with Lenses, Mirrors and Polarized Light. Guidelines for creating effective ILDs will also be discussed.

Do students learn optics concepts from ILDs? Here we report on assessments of learning gains for the Image Formation with Lenses ILD sequence. Students in the algebra-trigonometry-based general physics course at the University of Oregon had only a 20\% normalized learning gain on our physics education research-based Light and Optics Conceptual Evaluation after all traditional instruction on image formation. With just one additional lecture consisting of this $I L D$ sequence, their learning gain from the pre-test was $80 \%$. In addition, the last question on the test shows the real image of an arrow formed by a lens, with two (non-principal) rays from the bottom of the arrow and two (non-principal) rays from the top of the arrow incident on the lens. (See Figure 1). Students are asked to continue these four rays through the lens to illustrate how the image is formed by the lens. While after traditional instruction, only $33 \%$ were able to continue these rays correctly, after experiencing the ILD sequence, $76 \%$ completed this exercise correctly.

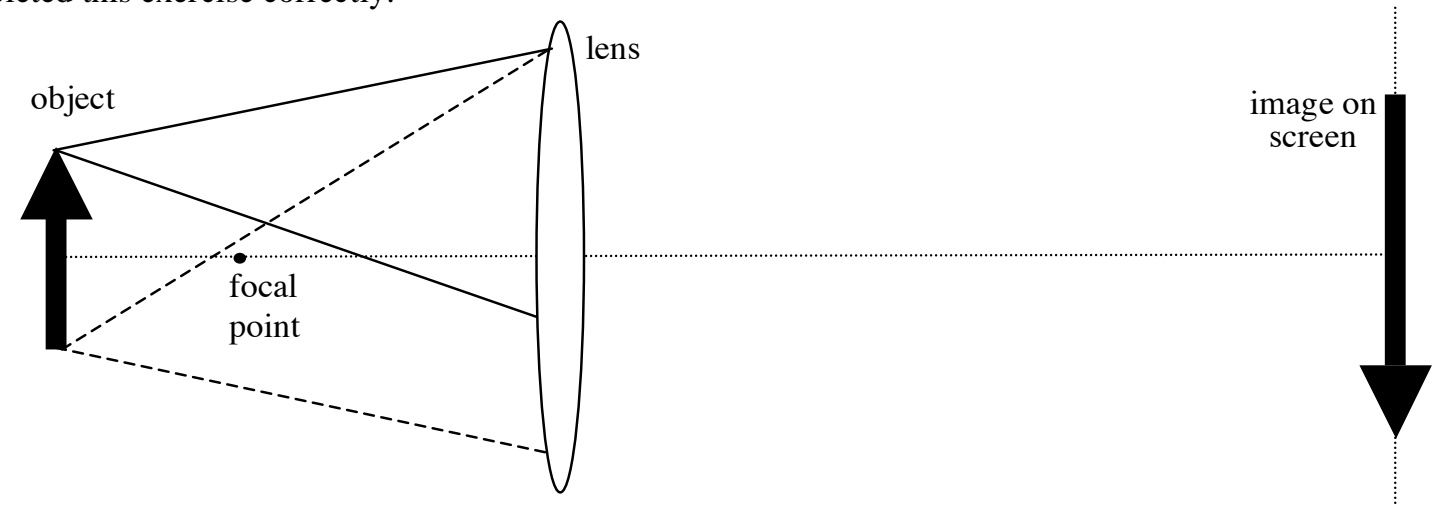

Figure 1: Diagram from last question on the Light and Optics Conceptual Evaluation

\section{Optics Magic Tricks}

A series of Optics Magic Tricks that introduce students to basic optics concepts will also be presented. There are a total of 10 tricks, illustrating concepts in reflection and refraction, image formation with mirrors, total internal reflection, light scattering and polarization. ${ }^{7}$ These include The Reappearing Test Tube, an illustration of the importance of index of refraction for seeing transparent media, Carbon to Silver, an example of total internal refraction and Candle Burning Under Water, an illustration of image formation with a plane mirror. All are presented in a highly active way through the use of optics conceptual learning questions. The remainder of this paper will give details on preparation and presentation of the tricks. 


\subsection{The Reappearing Test Tube}

Objective: To understand the importance of a difference in optical properties (indexes of refraction) of transparent media in the reflection and refraction of light.

Equipment and Supplies: $2600 \mathrm{ml}$ clear glass beakers, 3 small clear Pyrex culture or test tubes, hammer or block of wood, envelope, vegetable oil or light and heavy mineral oils. (In Europe, paraffin oil and Duraglas test tubes will work and may be more readily available.)

Preparation Notes: The index of refraction of vegetable oil is very close to that of Pyrex. Or, you can mix the mineral oils to match the index of refraction more closely to the index of the tubes. Fill one beaker with this "magic fluid" and completely submerge one or two tubes in it. You should not be able to see the submerged tubes. Fill the other beaker with water. It is best if the lights are dimmed somewhat, and a white background behind the beakers helps to make the tubes invisible.

Presentation Notes: Take a dry tube and place it in the envelope. Smash it with the hammer or block of wood. Ask a volunteer to look into the envelope to verify that the tube is smashed. Tell the students that the beaker contains a magic fluid that will repair the tube. Drop the pieces of the tube into the beaker with the mineral oil. Say some "magic" word(s) and/or wave a magic wand. Then reach in and pull out a whole tube! For laughs, you can say that this trick is even more amazing, and pull out a second tube!

The Optics Learning Questions for this trick are shown in Figure 2. Break down the class into small groups, and ask the groups to discuss these questions. Then ask for volunteer(s) to answer them.

\section{Reappearing Test Tube Learning Questions}

1. How do you think that the test tube was made to reappear?

. Why can you see a test tube in air or in water, but not in the magic fluid? What is special about the magic fluid?

3. What property of transparent media determines whether reflection takes place at the boundary between them? What has to be true about this property for the two materials in order for reflection to take place?

4. What about the light that is transmitted through the test tube? How is it affected when the test tube is in the magic fluid and when the test tube is in air?

Figure 2: Optics Learning Questions for Reappearing Test Tube.

Explanation: Clear objects only reflect and refract light when they are in a medium with different optical properties (a different index of refraction). Since the "magic fluid" has the same index as the tube, no light is reflected by the submerged tube to your eyes. Therefore, you cannot see it. Show that you can see the tube in air because air has a different index than the tube. Also show that you can see it in water. Take a dry tube and submerge it open side up so that the vegetable (or mineral oil) flows over the rim. It will appear to the students to disappear from the bottom up! Some students have even described that it seems to disappear in a flash! 


\subsection{Candle Burning Under Water}

Objective: To explore the properties of a virtual image formed by a plane mirror.

Equipment and Supplies: Candle, matches, container of water, $600 \mathrm{ml}$ clear glass beaker, large (at least $60 \mathrm{~cm} \times 60$ $\mathrm{cm}$ ) acrylic or glass sheet supported vertically, black (or other dark colored) cloth large enough to cover the sheet.

Preparation Notes: Place the candle in front of the acrylic sheet facing the students, and the beaker a distance behind the sheet so that the image of the candle as seen from in front of the acrylic sheet appears to be in the center of the beaker. The black cloth should be over the acrylic sheet. It is best if the lights are dimmed.

Presentation Notes: Light the candle, and pretend to light a candle in the beaker behind the sheet as well. Remove the black cloth. Say the magic word(S), and fill the beaker behind the sheet with water. It will appear to students that there is a candle burning under water in the beaker.

Use small groups and the Optics Learning Questions in Figure 3. Ask for volunteer(s) to explain how the trick works.

\section{Candle Under Water Learning Questions}

1. How do you think that it was possible for the candle to burn under water?

2. Describe the image of the candle formed by the glass (acrylic) mirror. Is the image real or virtual? Define both of these types of images.

3. Is the image upright or inverted compared to the candle? Compare the size of the image to the candle.

4. Compare the "handedness" of the image in a plane mirror to that of the object. That is if the object is a right hand, is the image a right or left hand?

5. What property of the glass (or acrylic) allows the sheet to act as a mirror?

\section{Figure 3: Optics Learning Questions for Candle Under Water}

Explanation: The acrylic sheet acts as a plane mirror, forming a virtual image of the lit candle in the beaker behind the sheet. Even though the sheet only reflects about $4-8 \%$ of the light from the candle, this is enough to have a clear, and somewhat convincing image. 


\subsection{Candle Appearing in a Box}

Objective: To explore the properties of a real image formed by a concave mirror.

Equipment and Supplies: Small candle (about $2 \mathrm{~cm}$ in diameter and $4 \mathrm{~cm}$ tall), large, front-surface, concave mirror (around $150 \mathrm{~cm}$ radius of curvature) with holder that allows fairly precise vertical and horizontal adjustment, wooden box about $30 \mathrm{~cm}$ high with $15 \mathrm{~cm}$ x $15 \mathrm{~cm}$ cross-section with three sides hinged so that they open to $90^{\circ}$.

Preparation Notes: Put the box on the table with the long dimension vertical, and with one of the opening sides facing the students and one facing away. (This demonstration will only work in this form with a small group of students. For larger groups, it is possible to use a video camera to project the image formed on a monitor or screen.) Place the candle on top of the box. With the sides of the box open, set up the mirror so that it forms a real, inverted image of the candle inside the box, and just below the real candle. (The distance of the mirror from the candle should equal the radius of curvature of the mirror-twice the focal length.) Close the sides of the box. It is best if the lights are dimmed.

Presentation Notes: Light the candle. Open the side of the box facing the students and show them that there is no candle inside. Close the side, say the magic word(s) and then open both sides. The inverted image of the candle will appear inside the box.

Ask for volunteer(s) to explain how the trick works.

Explanation: The concave mirror forms a real, inverted image at the location of the candle, but slightly displaced below. It looks like there is really a candle there! It is at the same location as the candle because the distance of the candle from the mirror equals the radius of the mirror (twice the focal length of the mirror). Contrast this image to the one formed by a plane mirror in the previous demonstration. Is it real or virtual? How do you know? (Put a piece of paper where the image is formed, and note that rays of light are really focused to that location, unlike the virtual image from the plane mirror in which no rays are focused behind the mirror where the image is formed.)

\subsection{The Magic of Spoons}

Objective: To compare the real image formed by a concave mirror to the virtual image formed by a convex mirror.

Equipment and Supplies: For a large class, it is best to make a large "spoon" from a large concave or convex mirror with no backing (both concave and convex sides reflect). Attach a handle to the mirror. The size of the mirror should be appropriate to the size of the class. The focal length should be small enough so that the students are much further away than the focal point. For small group(s) of students, individual real spoons that are polished enough to reflect an image work fine.

Presentation Notes: Hold up the "spoon" with the concave side facing the class (or have the students observe their images in the concave side of the spoons). Have them note whether the image is upright or inverted. (The image will be real and inverted.) Say the magic word(s), and flip the spoon so that they are looking into its convex side. Have them note that the image is now upright.

Ask for volunteer(s) to explain how the trick works.

Explanation: The object (student's face) is outside the focal point of the concave mirror. Therefore, the image formed is real and inverted. A convex mirror can only form a virtual upright image, and therefore, this is what is seen when the "spoon" is flipped around.

\subsection{Demonstration 5: Coal to Silver}

Objective: To observe total internal reflection at the interface between two transparent media.

Equipment and Supplies: Ball (about $5 \mathrm{~cm}$ in diameter) made of non-flammable/non-melting material mounted on the end of a rod and covered with soot (carbon) from a candle flame, $600 \mathrm{ml}$ beaker of water.

Preparation Notes: Light the candle and rotate the ball in the flame until the ball is covered all over with soot. Fill the beaker with water. 
Presentation Notes: Tell the students that while the alchemists tried to turn lead into gold, you have discovered how to turn coal into silver. Say the magic word(s) and submerge the ball in the water. The students will see light reflected off the surface of the ball, and it will appear to have a shiny silver-like surface.

Use small groups and the Optics Learning Questions in Figure 4. Ask for volunteer(s) to explain how the trick works.

Explanation: A layer of air is trapped around the surface of the ball by the carbon layer. Therefore, light from outside incident on this layer can be totally internally reflected back into the water and back to the students' eyes. It appears that the light is being reflected from the "shiny" surface of the ball. Total internal reflection takes place when light is incident from a medium with a larger index of refraction (water) on a medium with a smaller index of refraction (air layer).

\section{Carbon to Silver Learning Questions}

1. How do you think that the carbon ball was made to appear like silver?

2. What could cause the carbon ball to become a reflecting surface when it is submerged in water?

3. Compare the index of refraction of the air layer around the ball to the index of refraction of the water. What name is given to the reflection at this air layer?

4. Why is the surface of the ball not a "perfect" mirror?

Figure 4: Optics Learning Questions for Carbon to Silver

\subsection{Falling Laser Beam}

Objective: To observe total internal reflection at the interface between two transparent media-the mechanism of fiber optics.

Equipment and Supplies: Clear glass or acrylic container filled with water with a small (about 5-10 $\mathrm{mm}$ ) hole on one side near the bottom sealed by a stopper, laser, trough or pan on floor to catch water stream, blackboard eraser with chalk on it.

Preparation Notes: The laser is aligned so that it can be shined from the other side of the container through the water incident on the stopper from inside the water. 
Presentation Notes: Shine the laser across the room, and hit the eraser with your hand to suspend chalk dust in the laser beam. Note that the beam goes in a straight line. Then shine the laser on the stopper as described above. Say the magic word(s) and remove the stopper. The water streams out along a curved path, and the laser beam follows this path, apparently falling downward with the water.

Use small groups and the Optics Learning Questions in Figure 5. Ask for volunteer(s) to explain how the trick works.

\section{Falling Laser Beam Learning Questions}

1. What caused the laser beam to curve around and stay within the water stream?

2. Compare the index of refraction of the water to that of the air around it.

3. What name is given to the reflection of the light at the surface of the water stream?

4. What practical devices work on the same principle?

Figure 5: Optics Learning Questions for Falling Laser Beam

Explanation: The laser beam within the stream of water is incident on the interface between water and air, from higher index of refraction to lower index of refraction. Since the light is incident at an angle larger than the critical angle, the beam is totally internally reflected back into the water and seems to be trapped. This is the same mechanism that is exploited in fiber optics.

\subsection{Bouncing Laser Beam}

Objective: To observe curving of light in a medium with a continuously variable index of refraction-the mechanism of an oasis or mirage.

Equipment and Supplies: Acrylic trough about $15 \mathrm{~cm}$ deep, $5 \mathrm{~cm}$ wide and $75 \mathrm{~cm}$ long, thick corn syrup (e.g., Karo Syrup), laser, blackboard eraser with chalk on it.

Preparation Notes: At least one hour before the demonstration, pour the syrup into the trough to a depth of about 4 $\mathrm{cm}$. Pour water slowly to a depth of about $4 \mathrm{~cm}$ above the syrup. Mix carefully with a stirring rod so that the layers partially mix, but not enough so that they totally mix. Let stand for awhile. The idea is to get an index of refraction gradient with the smallest index (just water) at the top and the largest index (just syrup) at the bottom.

Presentation Notes: Shine the laser across the room, and hit the eraser with your hand to suspend chalk dust in the laser beam. Note that the beam goes in a straight line. Say the magic word(s), and then set up the laser so that it is incident on the trough through the $15 \mathrm{~cm}$ x $5 \mathrm{~cm}$ face, near the top of the fluid mixture. You may need to aim the beam down slightly. You should see the beam curve downward, reflect off the bottom, curve downward again 
(forming a half loop) after it reflects from the bottom, etc., etc. In other words, you should see several half loops of the beam, like a bouncing ball.

Ask for volunteer(s) to explain how the trick works.

Explanation: The index of refraction of the fluid increases from top to bottom. As the beam moves through this changing index, instead of refracting sharply at a surface, it bends slowly downward. When it hits the bottom, it is reflected upward again. It continues to bend, finally reaching an angle greater than the critical angle, and reflecting back down again, continuing to bend until it hits the bottom again.

4.8 Colors from a Magic Solution

Objective: To observe one of the manifestations of optical rotation.

Equipment and Supplies: Thick corn syrup (e.g., Karo Syrup), 600-1000 ml glass or clear plastic jar with sealable lid, sheet of Polaroid, slide projector, Polaroid slide, opaque slide, transparent slide and screen.

Preparation Notes: Cut the sheet of Polaroid so that it fits inside the jar and covers one half of the surface of the jar (half cylindrical shape). Place the Polaroid inside the jar against the surface. Fill the jar with the corn syrup, and then seal the jar with the lid. (This jar can be used over and over again.) Cut another piece of Polaroid the size of a slide for the projector. Place the three slides in the projector in this order: transparent, opaque, Polaroid. The axis of the Polaroid slide should be at $90^{\circ}$ with the axis of the Polaroid in the jar with the jar vertical. Set up the projector so that it will shine through the jar and either be focused onto the screen or shined into the students' eyes.

Presentation Notes: With the transparent slide in place, shine the projector through the jar with the Polaroid side facing the students. Rotate the jar around an axis perpendicular to the front face. Nothing happens. Then move the opaque slide into place. Say the magic word(s), and quickly move the Polaroid slide into place. Now rotate the jar as before, and the students will see different colors of light appear.

Ask for volunteer(s) to explain how the trick works.

Explanation: The corn syrup has the property that it can rotate the axis of polarization of incident polarized light. Initially the incident light is un-polarized, so the corn syrup has no net effect. The Polaroid in the jar (the analyzer) does nothing. When polarized light is shined through the jar, the corn syrup rotates the axis of polarization different amounts for different wavelengths of the incident white light. Depending on the angle of the analyzer, a different color is passed through and seen.

\subsection{Appearing Message}

Objective: To observe the effect of bi-refringence.

Equipment and Supplies: Large sheet of Polaroid (e.g., $25 \mathrm{~cm}$ x $25 \mathrm{~cm}$ ), thin sheet of plastic (e.g., overhead transparency), slide projector, Polaroid slide, opaque slide and transparent slide.

Preparation Notes: Cut letters of a message out from the thin plastic sheet. ("OOH, AAH" is a fun message to use!) Tape them to the back of the large sheet of Polaroid. Cut a piece of Polaroid the size of a slide for the projector. Place the three slides in the projector in this order: transparent, opaque, Polaroid. The axis of the Polaroid slide should be at $90^{\circ}$ with the axis of the Polaroid sheet. Set up the projector so that it will shine through the large sheet of Polaroid towards the class, with the Polaroid facing the class and the letters behind it.

Presentation Notes: With the transparent slide in place, shine the projector through the Polaroid sheet toward the students, with the letters on the projector side. Nothing happens. Then move the opaque slide into place. Say the magic word(s), and quickly move the Polaroid slide into place. Now the letters appear.

Ask for volunteer(s) to explain how the trick works.

Explanation: The plastic sheet material is bi-refringent and does not have a completely uniform thickness. It rotates the axis of polarization of the Polarized light by different amounts depending on its thickness at different spots. 
Some of this light that has its axis rotated to a different angle than $90^{\circ}$ goes through the Polaroid sheet (the analyzer), so the students see the message.

\subsection{The Sunset}

Objective: To observe polarization by scattering, and how scattering produces the blue sky and the colors of the sunset.

Equipment and Supplies: Sheet of Polaroid (e.g., $15 \mathrm{~cm} \mathrm{x} 15 \mathrm{~cm}$ ), $1800 \mathrm{ml}$ clear plastic or glass beaker 3/4 filled with water, $40 \mathrm{ml}$ saturated sodium thiosulfate solution, $25 \mathrm{ml}$ concentrated hydrochloric acid, stirring rod, projector and screen. (Alternatively, drops of milk can be added to the water instead of using the sodium thiosulfate and acid. However, the sodium thiosulfate and acid produce a more dramatic effect.)

Preparation Notes: Set up the projector so that it shines through the beaker onto the screen. Just before lecture, add the sodium thiosulfate to the water in the beaker and stir well. Have the acid ready to add to the beaker, and the Polaroid ready to hold in front of the beaker (between the beaker and the students).

Presentation Notes: Turn on the projector, and tell the students it represents the sun. The water in the beaker represents the sky. Tell them that you are going to produce a sunset in class. Say the magic word(s), add the acid to the beaker and quickly stir. Shortly, bluish light will be scattered out of the side of the beaker. If you rotate the Polaroid, it will be apparent that this light is partially polarized. The light passing through the beaker to the screen will at first appear yellow, and then redder and redder.

Ask for volunteer(s) to explain how the trick works.

Explanation: The acid precipitates tiny particles of sodium in the water. These particles scatter the light out of the sides of the beaker. The scattering process is more effective the shorter the wavelength. Therefore, blue light is scattered more than yellow or red. At low concentrations of sulfur, the light that gets through to the screen is yellow. As time passes and the concentration increases, the color of the light passing through becomes redder.

In the sky, the blue scattered light is what we see during the day. (Actually, violet is scattered more effectively, but our eyes are much less sensitive to violet than to blue.) When the sun is setting, however, we are looking directly at the sun. Rather than seeing the scattered light, we are looking at the light left behind after scattering by a relatively thick layer of the atmosphere, and this is reddish in color.

\section{Conclusions}

All of these curricula have been used successfully by the author in his introductory college level physics course, and recently in a series of Active Learning in Optics and Photonics (ALOP) in developing countries (presented to date in Ghana, Tunisia, Morocco and India, and planned for Tanzania, Brazil and Mexico). These have been sponsored by UNESCO, OSA, ICTP and SPIE. More details on the ALOP project will be presented in a separate paper, "Active Learning in Optics and Photonics: Achievements and Outcomes to Date," with principal author M. Alarcon.

\section{References}

(1) David R. Sokoloff and Ronald K. Thornton, Interactive Lecture Demonstrations (Hoboken, NJ, John Wiley and Sons, 2004).

(2) David R. Sokoloff and Ronald K. Thornton, "Using Interactive Lecture Demonstrations to Create an Active Learning Environment," The Physics Teacher 35: 6, 340 (1997).

(3) Ronald K. Thornton and David R. Sokoloff, "Assessing Student Learning of Newton's Laws: The Force and Motion Conceptual Evaluation and the Evaluation of Active Learning Laboratory and Lecture Curricula," American Journal of Physics 66, 338-352 (1998).

(4) David R. Sokoloff, Ronald K. Thornton and Priscilla W. Laws, "RealTime Physics: Active Learning Labs Transforming the Introductory Laboratory," accepted for publication, Eur. J. of Phys., 2007.

(5) David R. Sokoloff, Ronald K. Thornton and Priscilla W. Laws, RealTime Physics Module 1: Mechanics, Module 2: Heat and Thermodynamics, Module 3: Electric Circuits and Module 4: Light and Optics (Hoboken, NJ, John Wiley and Sons, 2004).

(6) Thornton, R.K. and Sokoloff, D.R., "RealTime Physics: Active Learning Laboratory," in The Changing Role of the Physics Department in Modern Universities, Proceedings of the International Conference on Undergraduate Physics Education, 1101-1118 (American Institute of Physics, 1997).

(7) Here are some resources from which these demonstrations were collected: Paul G. Hewitt, Instructor's Manual to Conceptual Physics, $3^{\text {rd }}$ Ed. (Boston, Little, Brown and Co., 1977), Richard Sutton, Demonstration Experiments in Physics (New York, McGraw-Hill, 1938), G.D. Freier and F.J. Anderson, A Demonstration Handbook for Physics (College Park, MD, American Association of Physics Teachers, 1981), and T. Kallard, Exploring Laser Light (College Park, MD, American Association of Physics Teachers, 1982). 